PROCEEDINGS OF THE

AMERICAN MATHEMATICAL SOCIETY

Volume 134, Number 2, Pages 419-426

S 0002-9939(05)08382-6

Article electronically published on September 20, 2005

\title{
ANGULAR SELF-INTERSECTIONS FOR CLOSED GEODESICS ON SURFACES
}

\author{
MARK POLLICOTT AND RICHARD SHARP
}

(Communicated by Michael Handel)

\begin{abstract}
In this note we consider asymptotic results for self-intersections of closed geodesics on surfaces for which the angle of the intersection occurs in a given arc. We do this by extending Bonahon's definition of intersection forms for surfaces.
\end{abstract}

\section{INTRODUCTION}

In recent years, many authors have considered the self-intersection of geodesics $[\mathrm{BS}, \mathrm{Bo1}, \mathrm{Po}, \mathrm{La}$. In this note we consider the problem of estimating the number of self-intersections for which the angle of intersection lies in a given arc. Let $V$ be a compact surface with negative curvature, and let $\gamma$ denote a closed geodesic on $V$ of length $l(\gamma)$. It is a classical result of Huber [Hu1 and Margulis Ma that the number $N(T)$ of closed geodesics of length at most $T$ satisfies the asymptotic formula $N(T) \sim e^{h T} / h T$, as $T \rightarrow+\infty$, i.e., the ratio of the two sides converges to one. (Here $h>0$ denotes the topological entropy of the geodesic flow over $V$.) In the case where $V$ has constant curvature, Huber Hu2 obtained the stronger estimate $N(T)=\operatorname{li}\left(e^{h T}\right)\left(1+O\left(e^{-\delta T}\right)\right)$, for some $\delta>0$, while for variable curvature this was established only in recent years [PS], Do].

For $0 \leq \theta_{1}<\theta_{2} \leq \pi$ we let $i_{\theta_{1}, \theta_{2}}(\gamma)$ denote the number of self-intersections of the closed geodesic $\gamma$ such that the absolute value of the angle of intersection lies in the interval $\left[\theta_{1}, \theta_{2}\right]$.

Theorem 1. Given $0 \leq \theta_{1}<\theta_{2} \leq \pi$, there exists $I=I\left(\theta_{1}, \theta_{2}\right)$ and $\delta>0$ such that, for any $\epsilon>0$,

$$
\#\left\{\gamma: l(\gamma) \leq T, \frac{i_{\theta_{1} \theta_{2}}(\gamma)}{l(\gamma)^{2}} \in(I-\epsilon, I+\epsilon)\right\}=\operatorname{li}\left(e^{h T}\right)\left(1+O\left(e^{-\delta T}\right)\right) .
$$

If $\theta_{1}=0$ and $\theta_{2}=\pi$, then $i_{0, \pi}(\gamma)$ is simply the total number of self-intersections of $\gamma$. In this particular case, Anantharaman An] has observed this result with $I(0, \pi)=i(\tilde{m}, \tilde{m}) / 2$, where $\tilde{m}$ is the transverse measure associated to the measure of maximal entropy for the geodesic flow over $V$ and $i(\cdot, \cdot)$ is the intersection form introduced by Bonahon Bo1] (see also $\mathrm{Ot}$ ). Our proof follows the same basic approach as that of $\mathrm{La}$ and $[\mathrm{An}$, in that we apply a standard large deviation result

Received by the editors October 15, 2003 and, in revised form, September 4, 2004.

2000 Mathematics Subject Classification. Primary 37C27, 37D20, 37D35, 37D40.

The second author was supported by an EPSRC Advanced Research Fellowship.

(C)2005 American Mathematical Society 
for closed orbits. The distinction is that we need to introduce two new ingredients: the angular intersection bundle and the associated angular intersection form. This allows us to restrict to counting the self-intersections of geodesics to those whose angle of intersection lies in a given interval.

We can interpret the constant $I\left(\theta_{1}, \theta_{2}\right)$ in terms of an angular intersection form, generalising $i(\cdot, \cdot)$. For a surface of constant negative curvature, it can be easily described explicitly, and we have the formula

$$
I\left(\theta_{1}, \theta_{2}\right)=\frac{1}{2 \pi \operatorname{Area}(V)} \int_{\theta_{1}}^{\theta_{2}} \sin \theta d \theta .
$$

In particular, using Area $(V)=4 \pi(g-1)$, where $g \geq 2$ is the genus of $V$, we can verify a heuristic estimate of Sieber and Richter [SR that

$$
\sum_{l(\gamma) \leq T} i_{\theta_{1}, \theta_{2}}(\gamma) \sim\left(\int_{\theta_{1}}^{\theta_{2}} \sin \theta d \theta\right) \frac{T^{2}}{8 \pi^{2}(g-1)} N(T) .
$$

Quantum Chaos provides an interesting motivation for this result associated to the study of pair correlations of energy levels $[\mathrm{Ke}]$. The associated form factor, or Fourier transform, leads to correlations of closed geodesics, and (0.1) is relevant to the leading term of the off-diagonal contribution.

We can also consider the distribution of the self-intersection points of the geodesic $\gamma$ on the surface $V$. We let $\mathcal{S}\left(\gamma ; \theta_{1}, \theta_{2}\right) \subset V$ denote the set of self-intersection points of $\gamma$ such that the absolute value of the angle of intersection lies in the interval $\left[\theta_{1}, \theta_{2}\right]$. In particular, $i_{\theta_{1}, \theta_{2}}(\gamma)=\# \mathcal{S}\left(\gamma ; \theta_{1}, \theta_{2}\right)$. Given a continuous function $f$ : $V \rightarrow \mathbb{R}$, we let $A\left(f, \gamma ; \theta_{1}, \theta_{2}\right)$ denote the sum of $f$ over the points of self-intersection of $\gamma$ lying in $\mathcal{S}\left(\gamma ; \theta_{1}, \theta_{2}\right)$, i.e.,

$$
A\left(f, \gamma ; \theta_{1}, \theta_{2}\right)=\sum_{x \in \mathcal{S}\left(\gamma ; \theta_{1}, \theta_{2}\right)} f(x) .
$$

Theorem 2. Given $0 \leq \theta_{1}<\theta_{2} \leq \pi$, there exists $I_{f}=I\left(f ; \theta_{1}, \theta_{2}\right)$ and $\delta>0$ such that, for any $\epsilon>0$,

$$
\#\left\{\gamma: l(\gamma) \leq T, \frac{A\left(f, \gamma ; \theta_{1}, \theta_{2}\right)}{l(\gamma)^{2}} \in\left(I_{f}-\epsilon, I_{f}+\epsilon\right)\right\}=\operatorname{li}\left(e^{h T}\right)\left(1+O\left(e^{-\delta T}\right)\right)
$$

In particular, when $f=1$, Theorem 2 reduces to Theorem 1 .

Sections 1 and 2 contain preliminary material. Section 3 contains the proofs of Theorem 1 and Theorem 2.

\section{INTERSECTION BUNDLES AND FORMS}

1.1 The geodesic flow. Let $S V$ be the unit tangent bundle of $V$ and let $\pi: S V \rightarrow$ $V$ denote the canonical projection. For $x \in V$, we write $S_{x} V=\pi^{-1}(x)$. Consider the geodesic flow $\phi_{t}: S V \rightarrow S V$ on the unit tangent bundle of $V$. This is an example of a weak-mixing hyperbolic flow $\mathrm{As}$. There is a one-to-one correspondence between oriented closed geodesics on $V$ and periodic orbits for $\phi$. We shall write $h>0$ for the topological entropy of $\phi$ and, given a $\phi$-invariant probability measure $\mu$, we shall write $h(\mu)$ for the measure theoretic entropy of $\phi$ with respect to $\mu$. There is a unique invariant probability measure $m$, called the measure of maximal entropy, for which $h(m)=h$. 
We let $\mathcal{F}$ denote the foliation of $S V$ by $\phi$-orbits. Given any $\phi$-invariant finite measure $\mu$ (not necessarily a probability measure) we can consider the associated transverse measure $\tilde{\mu}$ for $\mathcal{F}$. The set of these transverse measures $\mathcal{C}=$ $\{\tilde{\mu}: \mu$ is a $\phi$-invariant measure $\}$ is called the space of currents. Each $\tilde{\mu} \in \mathcal{C}$ is normalized by the requirement that (locally) $\mu=\tilde{\mu} \times d t$, where $d t$ is one-dimensional Lebesgue measure along orbits in $\mathcal{F}$.

1.2 Bundles. Let $E=S V \oplus S V-\Delta$ be the Whitney sum of the bundle $S V$ with itself minus the diagonal $\Delta=\left\{(x, v, v): x \in V, v \in S_{x} V\right\}$, and let $p: E \rightarrow V$ denote the canonical projection. In particular, points of the four-dimensional vector bundle $E$ (with two-dimensional fibres) consist of triples $(x, v, w)$, where $x \in V$ and $v, w \in S_{x} V$. Let $p_{1}: E \rightarrow S V$ be defined by $p_{1}(x, v, w)=(x, v)$, and let $p_{2}: E \rightarrow S V$ be defined by $p_{2}(x, v, w)=(x, w)$. Following Bonahon Bo1, we consider the two transverse foliations (with two-dimensional leaves) of $E$ given by $\mathcal{F}_{1}=p_{1}^{-1}(\mathcal{F})=\mathcal{F}_{2}=p_{2}^{-1}(\mathcal{F})$.

Definition. Given $0 \leq \theta_{1}<\theta_{2} \leq \pi$, we define the angular intersection bundle $E_{\theta_{1}, \theta_{2}}$ by $E_{\theta_{1}, \theta_{2}}=\left\{(x, v, w) \in E: \angle v, w \in\left[\theta_{1}, \theta_{2}\right]\right\}$, where $0 \leq \angle v, w \leq \pi$ denotes the angle between the two vectors. This is a closed subbundle of $E$.

It is sometimes convenient to consider an equivalent geometric definition of the bundle $E_{\theta_{1}, \theta_{2}}$ in terms of pairs of geodesics on the universal cover $\widetilde{V}$ of $V$. In particular, consider the space $\mathcal{G}_{\theta_{1}, \theta_{2}}$ of pairs of oriented geodesics $\left(\widetilde{\gamma}_{1}, \widetilde{\gamma}_{2}\right)$ on $\widetilde{V}$ which intersect, at some point $\widetilde{x} \in \widetilde{V}$, say, and with an angle of intersection in the interval $\left[\theta_{1}, \theta_{2}\right]$. There is a natural identification $E_{\theta_{1}, \theta_{2}}$ with $\mathcal{G}_{\theta_{1}, \theta_{2}} / \pi_{1}(V)$, where the quotient is with respect to the diagonal action of the fundamental group (cf. Bo2]).

1.3 Intersection forms. Given currents $\tilde{\mu}, \tilde{\mu}^{\prime} \in \mathcal{C}$, we can take the lifts $\widehat{\mu}_{1}:=$ $p_{1}^{-1} \tilde{\mu}$ and $\widehat{\mu}_{2}^{\prime}:=p_{2}^{-1} \tilde{\mu}^{\prime}$, which are transverse measures to the foliations $\mathcal{F}_{1}$ and $\mathcal{F}_{2}$ for $E$, respectively. Bonahon defined the intersection form $i: \mathcal{C} \times \mathcal{C} \rightarrow \mathbb{R}^{+}$ to be the total mass of the $E$ with respect to the product measure $\widehat{\mu}_{1} \times \widehat{\mu}_{2}^{\prime}$, i.e., $i\left(\tilde{\mu}, \tilde{\mu}^{\prime}\right)=\left(\widehat{\mu}_{1} \times \widehat{\mu}_{2}^{\prime}\right)(E)$ Bo1. By analogy, we can define the following.

Definition. We define an angular intersection form $i_{\theta_{1}, \theta_{2}}: \mathcal{C} \times \mathcal{C} \rightarrow \mathbb{R}^{+}$to be the total mass of the $E_{\theta_{1}, \theta_{2}}$ with respect to the product measure $\widehat{\mu}_{1} \times \widehat{\mu}_{2}^{\prime}$, i.e., $i_{\theta_{1}, \theta_{2}}\left(\tilde{\mu}, \tilde{\mu}^{\prime}\right)=\left(\widehat{\mu}_{1} \times \widehat{\mu}_{2}^{\prime}\right)\left(E_{\theta_{1}, \theta_{2}}\right)$.

In the geometric picture, the space of currents corresponds to measures on $\mathcal{G}_{\theta_{1}, \theta_{2}} / \pi_{1}(V)$. For example, given a geodesic $\gamma$ on $V$, the associated measure is simply a finite sum of Dirac measures supported on the quotient of pairs of closed geodesics on $\widetilde{V}$ consisting of lifts of $\gamma$ and its $\pi_{1}(V)$ images (cf. Bo2]).

More generally, in order to study the spatial distribution of the intersection points, we can introduce a weighted form. Let $f: V \rightarrow \mathbb{R}$ be a continuous function.

Definition. We define the weighted angular intersection form $i_{f ; \theta_{1}, \theta_{2}}: \mathcal{C} \times \mathcal{C} \rightarrow \mathbb{R}^{+}$ to be the integral of $f \circ p$ restricted to $E_{\theta_{1}, \theta_{2}}$ with respect to the product measure $\widehat{\mu}_{1} \times \widehat{\mu}_{2}^{\prime}$, i.e., $i_{f ; \theta_{1}, \theta_{2}}\left(\tilde{\mu}, \tilde{\mu}^{\prime}\right)=\int f \circ p d\left(\widehat{\mu}_{1} \times \widehat{\mu}_{2}^{\prime}\right)$. 


\section{INTERSECTION FORMS AND CLOSED GEODESICS}

Given a closed geodesic $\gamma$, let $\mu_{\gamma}$ denote the unique invariant measure of total mass $l(\gamma)$ supported on the corresponding periodic orbit and let $\tilde{\mu}_{\gamma}$ be the corresponding transverse measures for the orbit foliation $\mathcal{F}$, which are normalized to be a finite sum of Dirac measures on transverse sections. We shall write $\widehat{\mu}_{\gamma, 1}=p_{1}^{-1} \tilde{\mu}_{\gamma}$ and $\widehat{\mu}_{\gamma, 2}=p_{2}^{-1} \tilde{\mu}_{\gamma}$.

Lemma 1. Let $\gamma$ and $\gamma^{\prime}$ be a pair of closed geodesics. Then:

(i) $\left(\widehat{\mu}_{\gamma, 1} \times \widehat{\mu}_{\gamma^{\prime}, 2}\right)\left(E_{\theta_{1}, \theta_{2}}\right)$ is equal to the number of intersections between $\gamma$ and $\gamma^{\prime}$ with angle between $\theta_{1}$ and $\theta_{2}$, and

(ii) given a continuous function $f: V \rightarrow \mathbb{R}, \int_{E_{\theta_{1}, \theta_{2}}} f \circ p d\left(\widehat{\mu}_{\gamma, 1} \times \widehat{\mu}_{\gamma^{\prime}, 2}\right)$ is equal to the summation of $f$ over the points of intersection of $\gamma$ and $\gamma^{\prime}$ with angle between $\theta_{1}$ and $\theta_{2}$.

Proof. The proof is modelled on [Bo1, p. 111]. We can choose flow boxes $B_{1}, B_{2} \subset$ $S V$ for $\phi$ such that, provided they are sufficiently small, for $v_{1} \in B_{1}$ and $v_{2} \in B_{2}$ the associated geodesic arcs intersect transversally at one point at most. We denote

$$
B_{1} \oplus B_{2}:=p_{1}^{-1}\left(B_{1}\right) \cap p_{2}^{-1}\left(B_{2}\right) \subset E .
$$

As in Bo1, one can see that $\left(\widehat{\mu}_{\gamma, 1} \times \widehat{\mu}_{\gamma^{\prime}, 2}\right)\left(B_{1} \oplus B_{2}\right)$ is precisely the number of times $\gamma$ crosses $\gamma^{\prime}$ with tangent vectors in $B_{1}$ and $B_{2}$, respectively. Covering $S V$ by a partition consisting of such flow boxes, and summing over those $B_{1} \oplus B_{2}$ which approximate $E_{\theta_{1}, \theta_{2}}$ gives an estimate to the number of times the geodesics intersect with angle in $\left[\theta_{1}, \theta_{2}\right]$, i.e., if $d(\cdot, \cdot)$ denotes the Hausdorff distance between closed subsets of $E$,

$$
\sum_{d\left(E_{\theta_{1}, \theta_{2}}, B_{1} \oplus B_{2}\right)<\epsilon}\left(\widehat{\mu}_{\gamma, 1} \times \widehat{\mu}_{\gamma^{\prime}, 2}\right)\left(B_{1} \oplus B_{2}\right)
$$

can be made arbitrarily close to $i_{\theta_{1}, \theta_{1}}\left(\tilde{\mu}_{\gamma}, \tilde{\mu}_{\gamma^{\prime}}\right)$ by choosing $\epsilon>0$ sufficiently small. By taking the size of the flow boxes arbitrarily small, the result follows.

For part (ii), it suffices to show that, for any small disk $D \subset V$, we have that $\left(\widehat{\mu}_{\gamma, 1} \times \widehat{\mu}_{\gamma^{\prime}, 2}\right)\left(E_{\theta_{1}, \theta_{2}} \cap p^{-1}(D)\right)$ is equal to the number of intersections between $\gamma$ and $\gamma^{\prime}$ lying inside $D$ and with angle between $\theta_{1}$ and $\theta_{2}$. However, this follows from the preceeding argument if we further restrict the summation to those $B_{1} \oplus B_{2}$ whose projections $\pi\left(B_{1}\right)$ and $\pi\left(B_{2}\right)$ approximate $D$.

As immediate consequences of the above lemma we have that the number of self-intersections of $\gamma$ with angle lying in the interval $\left[\theta_{1}, \theta_{2}\right]$ is given by $i_{\theta_{1}, \theta_{2}}(\gamma)=$ $\left(\widehat{\mu}_{\gamma, 1} \times \widehat{\mu}_{\gamma, 2}\right)\left(E_{\theta_{1}, \theta_{2}}\right) / 2$ and that $A\left(f, \gamma ; \theta_{1}, \theta_{2}\right)=\int_{E_{\theta_{1}, \theta_{2}}} f \circ p d\left(\widehat{\mu}_{\gamma, 1} \times \widehat{\mu}_{\gamma, 2}\right) / 2$.

The next result will show that $\tilde{\mu} \mapsto i_{\theta_{1}, \theta_{2}}(\tilde{\mu}, \tilde{\mu})$ is continuous in a neighbourhood of the transverse measure $\tilde{m}$ associated to the measure of maximal entropy $m$. Let $\widehat{m}_{1}=p_{1}^{-1} \tilde{m}$ and $\widehat{m}_{2}=p_{2}^{-1} \tilde{m}$.

Lemma 2. Let $\gamma_{n}$ be a sequence of closed geodesics and let $\mu_{\gamma_{n}} / l\left(\gamma_{n}\right)$ be the (normalised) probability measure on $S V$. Assume that $\mu_{\gamma_{n}} / l\left(\gamma_{n}\right)$ converges in the weak* topology to the measure of maximal entropy $m$. Then

(1) $\left(\widehat{\mu}_{\gamma_{n}, 1} \times \widehat{\mu}_{\gamma_{n}, 2}\right)\left(E_{\theta_{1}, \theta_{2}}\right) \rightarrow\left(\widehat{m}_{1} \times \widehat{m}_{2}\right)\left(E_{\theta_{1}, \theta_{2}}\right)$, as $n \rightarrow+\infty$; and

(2) given a continuous function $f: V \rightarrow \mathbb{R}, \int_{E_{\theta_{1}, \theta_{2}}}(f \circ p) d\left(\widehat{\mu}_{\gamma_{n}, 1} \times \widehat{\mu}_{\gamma_{n}, 2}\right)\left(E_{\theta_{1}, \theta_{2}}\right)$ $\rightarrow \int_{E_{\theta_{1}, \theta_{2}}}(f \circ p) d\left(\widehat{m}_{1} \times \widehat{m}_{2}\right)$, as $n \rightarrow+\infty$. 
Proof. We recall Bonahon's proof that $\left(\widehat{\mu}_{\gamma_{n}, 1} \times \widehat{\mu}_{\gamma_{n}, 2}\right)(E) \rightarrow\left(\widehat{m}_{1} \times \widehat{m}_{2}\right)(E)$, as $n \rightarrow+\infty$ [Bo1, pp. 112-114]. In his proof, he approximated $E-U$, where $U$ is a neighbourhood of the diagonal $\Delta$, by unions of sets $B_{1} \oplus B_{2}$, where $B_{1}$ and $B_{2}$ are sufficiently small flow boxes. He then showed that $\left(\widehat{\mu}_{\gamma_{n}, 1} \times \widehat{\mu}_{\gamma_{n}, 2}\right)\left(B_{1} \oplus B_{2}\right) \rightarrow$ $\left(\widehat{m}_{1} \times \widehat{m}_{2}\right)\left(B_{1} \oplus B_{2}\right)$, as $n \rightarrow+\infty$. By similarly approximating $E_{\theta_{1}, \theta_{2}}$ by unions $B_{1} \oplus B_{2}$, we see that part (1) holds.

For part (2), it suffices to show that, for any small disk $D \subset V$, we have that $\left(\widehat{\mu}_{\gamma_{n}, 1} \times \widehat{\mu}_{\gamma_{n}, 2}\right)\left(E_{\theta_{1}, \theta_{2}} \cap p^{-1}(D)\right) \rightarrow\left(\widehat{m}_{1} \times \widehat{m}_{2}\right)\left(E_{\theta_{1}, \theta_{2}} \cap p^{-1}(D)\right)$, as $n \rightarrow+\infty$. In the preceeding argument we may further restrict the union to those $B_{1} \oplus B_{2}$ whose projections $\pi\left(B_{1}\right)$ and $\pi\left(B_{2}\right)$ approximate $D$. This completes the proof.

\section{Proof of the theorems}

Theorems 1 and 2 will follow from a large deviation result for periodic orbit measures established by Kifer Ki]. Kifer's result is valid for any hyperbolic flow and so, in particular, for the geodesic flow $\phi_{t}: S V \rightarrow S V$.

Lemma 3 ([Ki]). Let $\mathcal{U}$ be an open neighbourhood of the measure of maximal entropy $m$ in the set of $\phi$-invariant probability measures on $S V$. Then

$$
\frac{1}{N(T)} \#\left\{\gamma: l(\gamma) \leq T \text { and } \mu_{\gamma} / l(\gamma) \notin \mathcal{U}\right\}=O\left(e^{-\delta T}\right)
$$

as $T \rightarrow+\infty$, where $\delta=\inf _{\nu \in \mathcal{U}^{c}}\{h-h(\nu)\}$.

In particular, if $m \in \mathcal{U}$, then $\delta>0$. In our context, Lemma 3 gives the following estimates.

Lemma 4. (1) Given $\epsilon>0$, there exists $\delta>0$ such that

$$
\begin{aligned}
\frac{1}{N(T)} \#\left\{\gamma: l(\gamma) \leq T \text { and } \mid l(\gamma)^{-2}\left(\widehat{\mu}_{\gamma, 1}\right.\right. & \left.\times \widehat{\mu}_{\gamma, 2}\right)\left(E_{\theta_{1}, \theta_{2}}\right) \\
& \left.-\left(\widehat{m}_{1} \times \widehat{m}_{2}\right)\left(E_{\theta_{1}, \theta_{2}}\right) \mid \geq \epsilon\right\}=O\left(e^{-\delta T}\right),
\end{aligned}
$$

as $T \rightarrow+\infty$; and

(2) Let $f: V \rightarrow \mathbb{R}$ be a continuous function. Given $\epsilon>0$, there exists $\delta>0$ such that

$$
\begin{aligned}
\frac{1}{N(T)} \#\left\{\gamma: l(\gamma) \leq T \text { and } \mid \frac{1}{l(\gamma)^{2}}\right. & \int_{E_{\theta_{1}, \theta_{2}}} f \circ p d\left(\widehat{\mu}_{\gamma, 1} \times \widehat{\mu}_{\gamma, 2}\right) \\
& \left.-\int_{E_{\theta_{1}, \theta_{2}}} f \circ p d\left(\widehat{m}_{1} \times \widehat{m}_{2}\right) \mid \geq \epsilon\right\}=O\left(e^{-\delta T}\right),
\end{aligned}
$$

as $T \rightarrow+\infty$.

Proof. To prove part (1), we cannot directly apply Lemma 3 with

$$
\mathcal{U}=\left\{\nu:\left|\left(\widehat{\nu}_{1} \times \widehat{\nu}_{2}\right)\left(E_{\theta_{1}, \theta_{2}}\right)-\left(\widehat{m}_{1} \times \widehat{m}_{2}\right)\left(E_{\theta_{1}, \theta_{2}}\right)\right|<\epsilon\right\},
$$

since, as the indicator function for $E_{\theta_{1}, \theta_{2}}$ is not continuous, this set is not open. The measure $m$ is known to be non-atomic, and so we can deduce that $\widehat{m}_{1} \times \widehat{m}_{2}$ is also non-atomic. In particular, we can choose continuous functions $\psi_{1} \leq \chi_{E_{\theta_{1}, \theta_{2}}} \leq \psi_{2}$ 
such that

$$
\int \psi_{2} d\left(\widehat{m}_{1} \times \widehat{m}_{2}\right)-\tau \leq\left(\widehat{m}_{1} \times \widehat{m}_{2}\right)\left(E_{\theta_{1}, \theta_{2}}\right) \leq \int \psi_{1} d\left(\widehat{m}_{1} \times \widehat{m}_{2}\right)+\tau,
$$

for some $0<\tau<\epsilon$, and we can then deduce the required bound by considering

$$
\begin{aligned}
\mathcal{U}^{\prime}= & \left\{\nu: \int \psi_{2} d\left(\widehat{\nu}_{1} \times \widehat{\nu}_{2}\right)-\int \psi_{2} d\left(\widehat{\nu}_{1} \times \widehat{\nu}_{2}\right)<\epsilon-\tau\right\} \\
& \cap\left\{\nu: \int \psi_{1} d\left(\widehat{\nu}_{1} \times \widehat{\nu}_{2}\right)-\int \psi_{1} d\left(\widehat{\nu}_{1} \times \widehat{\nu}_{2}\right)>-\epsilon+\tau\right\}
\end{aligned}
$$

and noting that

$$
\#\left\{\gamma: l(\gamma) \leq T \text { and } \mu_{\gamma} / l(\gamma) \notin \mathcal{U}\right\} \leq \#\left\{\gamma: l(\gamma) \leq T \text { and } \mu_{\gamma} / l(\gamma) \notin \mathcal{U}^{\prime}\right\} .
$$

Clearly, $m \in \mathcal{U}^{\prime}$, so $\delta>0$, as required.

If $f$ is non-negative, then the proof of part (2) is similar. By choosing continuous functions $\psi_{3}(x, v) \leq(f \circ p)(x) \chi_{E_{\theta_{1}, \theta_{2}}}(v) \leq \psi_{4}(x, v)$ such that

$$
\int \psi_{4} d\left(\widehat{m}_{1} \times \widehat{m}_{2}\right)-\tau \leq \int_{E_{\theta_{1}, \theta_{2}}} f \circ p d\left(\widehat{m}_{1} \times \widehat{m}_{2}\right) \leq \int \psi_{3} d\left(\widehat{m}_{1} \times \widehat{m}_{2}\right)+\tau,
$$

for some $0<\tau<\epsilon$, we can deduce the required bound by considering

$$
\begin{aligned}
\mathcal{U}^{\prime \prime}=\left\{\gamma: \frac{1}{l(\gamma)^{2}} \int \psi_{4} d\left(\widehat{\mu}_{\gamma, 1} \times \widehat{\mu}_{\gamma, 2}\right)-\int \psi_{4} d\left(\widehat{m}_{1} \times \widehat{m}_{2}\right)<\epsilon-\tau\right\} \\
\cap\left\{\gamma: \frac{1}{l(\gamma)^{2}} \int \psi_{3} d\left(\widehat{\mu}_{\gamma, 1} \times \widehat{\mu}_{\gamma, 2}\right)-\int \psi_{3} d\left(\widehat{m}_{1} \times \widehat{m}_{2}\right)>-\epsilon+\tau\right\}
\end{aligned}
$$

and noting that

$$
\#\left\{\gamma: l(\gamma) \leq T \text { and } \mu_{\gamma} / l(\gamma) \notin \mathcal{U}\right\} \leq \#\left\{\gamma: l(\gamma) \leq T \text { and } \mu_{\gamma} / l(\gamma) \notin \mathcal{U}^{\prime \prime}\right\} .
$$

Clearly, $m \in \mathcal{U}^{\prime \prime}$, so $\delta>0$, as required. To obtain the general case, consider the positive and negative parts of $f$ separately.

Proof of Theorem 1. Write $I\left(\theta_{1}, \theta_{2}\right)=i_{\theta_{1}, \theta_{2}}(\tilde{m}, \tilde{m}) / 2$ and recall that $i_{\theta_{1}, \theta_{2}}(\gamma)=$ $\left(\widehat{\mu}_{\gamma, 1} \times \widehat{\mu}_{\gamma, 2}\right)\left(E_{\theta_{1}, \theta_{2}}\right) / 2$. We can apply part (1) of Lemma 4 to deduce that, except for an exceptional set with cardinality of order $O\left(e^{(h-\delta) T}\right)$, the set of closed geodesics of length at most $T$ satisfy $\left|l(\gamma)^{-2} i_{\theta_{1}, \theta_{2}}(\gamma)-I\left(\theta_{1}, \theta_{2}\right)\right|<\epsilon$. Theorem 1 then follows by applying the asymptotic counting results described in the introduction.

Proof of Theorem 2. This is similar to the proof of Theorem 1. We can write $I\left(\theta_{1}, \theta_{2}, f\right)=i_{\theta_{1}, \theta_{2}, f}(\tilde{m}, \tilde{m}) / 2$ and denote $i_{\theta_{1}, \theta_{2}, f}(\gamma)=i_{\theta_{1}, \theta_{2}}(\gamma) A\left(f, \gamma ; \theta_{1} \theta_{2}\right)$. We can apply part (1) of Lemma 4 to deduce that, except for an exceptional set with cardinality of order $O\left(e^{(h-\delta) T}\right)$, the set of closed geodesics of length at most $T$ satisfies $\left|l(\gamma)^{-2} i_{\theta_{1}, \theta_{2}, f}(\gamma)-I\left(\theta_{1}, \theta_{2}, f\right)\right|<\epsilon$. Theorem 2 then follows by applying the asymptotic counting results described in the Introduction.

An expression for $I\left(\theta_{1}, \theta_{2}\right)=\left(\widehat{m}_{1} \times \widehat{m}_{2}\right)\left(E_{\theta_{1}, \theta_{2}}\right) / 2$ can be given fairly explicity. Let $m_{V}$ be the projection onto the surface of $m$. For each $x \in V$, let $m_{x}$ be the induced measure on the fibre $S_{x} V$. Note that $m_{x}$ cannot have atoms (for $m_{V}$-a.e. $x$ ), since otherwise invariance of the measure would imply the existence of closed orbits with non-zero $m$ measure. However, since the measure of maximal entropy $m$ is fully supported, such orbits would immediately contradict the ergodicity of $m$. 
Lemma 5. We can write

$I\left(\theta_{1}, \theta_{2}\right)=\int_{V} \int\left(\int_{\substack{\left\{\left(v_{1}, v_{2}\right) \in S_{x} V \times S_{x} V \\ \angle v_{1}, v_{2} \in\left[\theta_{1}, \theta_{2}\right]\right\}}}: \sin \left(\angle v_{1}, v_{2}\right) d\left(m_{x} \times m_{x}\right)\left(v_{1}, v_{2}\right)\right) d m_{V}(x)$, where $\angle v_{1}, v_{2}$ is the angle between the vectors $v_{1}, v_{2} \in S_{x} V$ in the same fibre.

Proof. The intersection form is defined in terms of the total mass of the angular intersection bundle with respect to a product measure. One can approximate this value by approximating the bundle $E_{\theta_{1}, \theta_{2}}$ by the unions of small sets of the form $B_{1} \oplus B_{2}=p_{1}^{-1}\left(B_{1}\right) \cap p_{2}^{-1}\left(B_{2}\right)$ for pairs of flow boxes $B_{1}, B_{2}$ in $S V$ whose projections onto $V$ intersect transversely at a single point. By choosing the pairs of flow boxes $B_{1}, B_{2}$ sufficiently small, we can choose the angle of intersection of any geodesic arcs associated to $B_{1}$ and $B_{2}$, respectively, to be arbitrarily close to a constant $\theta$, say. The mass with respect to the product measure of the small set $B_{1} \oplus B_{2}$ is now particularly easy to estimate. It is given by the usual formula for the area of a parallelogram (height $\times$ base $\times \sin \theta$ ). Finally, we can take the union over all such sets $B_{1} \oplus B_{2}$ in the approximation to $E_{\theta_{1}, \theta_{2}}$. The estimate then follows by approximation.

In the particular case of constant curvature, one easily sees that the expression in Lemma 5 reduces to $(0.1)$.

\section{REFERENCES}

[An] N. Anantharaman, Distribution of closed geodesics on a surface, under homological constraints, preprint, 1999.

[As] D. Anosov, Geodesic flows of closed Riemann manifolds with negative curvature, Proceedings of the Steklov Institute of Mathematics, Vol. 90, Amer. Math. Soc., Providence, RI, 1969. MR0242194 (39:3527)

[BS] J. Birman and C. Series, Geodesics with bounded intersection number on surfaces are sparsely distributed, Topology 24 (1985), 217-225. MR0793185 (87f:57012)

[Bo1] F. Bonahon, Bouts des variétés hyperboliques de dimension 3, Annals of Math. 124 (1986), 71-158. MR0847953 (88c:57013)

[Bo2] F. Bonahon, The geometry of Teichmüller spaces via geodesic currents, Invent. Math. 92 (1988), 139-162. MR0931208 (90a:32025)

[Do] D. Dolgopyat, On statistical properties of geodesic flows on negatively curved surfaces, Ph.D. thesis, Princeton, 1997.

[Hu1] H. Huber, Zur analytischen Theorie hyperbolischer Raumformen und Bewegungsgruppen, Math. Ann. 138 (1959), 1-26. MR0109212 (22:99)

[Hu2] H. Huber, Zur analytischen Theorie hyperbolischer Raumformen und Bewegungsgruppen, II, Math. Ann. 142 (1961), 385-398. MR0126549 (23:A3845)

[Ke] J. Keating, Periodic orbits, spectral statistics, and the Riemann zeros, Supersymmetry and trace formulae (I. Lerner, J. Keating and D. Khmelnitskii, eds.), Kluwer, New York, 1999, pp. 1-15.

[Ki] Y. Kifer, Large deviations, averaging and periodic orbits of dynamical systems, Comm. Math. Phys. 162 (1994), 33-46. MR.1272765 (95b:58091)

[La] S. Lalley, Self-intersections of closed geodesics on a negatively curved surface: statistical regularities, Convergence in ergodic theory and probability (Columbus, OH, 1993), Ohio State Univ. Math. Res. Inst. Publ., 5, de Gruyter, Berlin, 1996, pp. 263-272. MR1412610 (97h:58103)

[Ma] G. Margulis, Certain applications of ergodic theory to the study of manifolds of negative curvature, Functional Anal. Appl. 3 (1969), 89-90. MR0257933(41:2582)

[Ot] J.-P. Otal, Le théorème d'hyperbolisation pour less variétés fibreés de dimension 3, Asterisque 235 (1996), 1-159. MR1402300 (97e:57013) 
[Po] M. Pollicott, Asymptotic distribution of closed geodesics, Israel J. Math. 52 (1985), 209-224. MR:0815810 (87g:58105)

[PS] M. Pollicott and R. Sharp, Exponential error terms for growth functions on negatively curved surfaces, Amer. J. Math. 120 (1998), 1019-1042. MR1646052 (99h:58148)

[SR] M. Sieber and K. Richter, Correlations between periodic orbits and their rôle in spectral statistics, Physica Scripta T90 (2001), 128-133.

Department of Mathematics, Manchester University, Oxford Road, Manchester M13 9PL, UNiTED Kingdom

Current address: Mathematics Institute, University of Warwick, Coventry CV4 7AL, United Kingdom

Department of Mathematics, Manchester University, Oxford Road, Manchester M13 9PL, UNited Kingdom 